\title{
STRATÉGIES ET ENJEUX DE LA "DÉPOLITISATION PAR LE LANGAGE”' DANS UN CORPUS DE PRESSE ACTUELLE
}

\author{
Roselyne Koren ${ }^{2}$
}

"Devoir d'irrespect", "contre-pouvoir" politique, exercice d'une "fonction critique", telles sont les dénominations que l'on trouve, entre autres, sous la plume des journalistes en France quand ils définissent la perception qu'ils ont de leur rôle. C'est à eux qu'il incomberait de résister aux manipulations du pouvoir exécutif et à ses tentatives d'intimidation. Leur engagement serait donc d'ordre éthique et non pas idéologique : il s'agit d'informer au nom de valeurs comme la vérité, la justice et la solidarité et non pas à des fins militantes. La politique est au centre des préoccupations de l'écriture de presse, mais les journalistes ne s'autorisent à prendre la parole que dans le cadre du politique entendu comme "lieu du projet collectif, du débat sur le possible, de l'engagement au service de la cité, de la lutte pour le bien commun"3.

\footnotetext{
1 J'emprunte cette dénomination à François Brune, Manière de voir Le Monde diplomatique 27, août 1995, "Médias et contrôle des esprits", p. 22.

2 Université Bar-Ilan, Israël.

3 Ibid.
}

Recherches en communication, $\mathrm{n}^{\circ} 20$ (2003). 
La sincérité des journalistes qui tiennent ce genre de discours ne fait pas de doute, mais cela ne signifie pas automatiquement qu'il faille renoncer à problématiser l'application des principes énoncés. La presse écrite actuelle est-elle à l'abri de la pensée unique et des langues de bois qu'elle est censée démasquer? Contribue-t-elle à la critique des institutions politiques et de l'immobilisme et donc à la politisation de l'opinion publique ou au contraire à sa dépolitisation ?

L'analyse du métadiscours sur la polémique et sur les modalités de la "guerre" des mots, dans un corpus d'une soixantaine d'articles" révèle que la balance penche du côté de la dépolitisation. Ce point de vue n'est pas nouveau : Jean-François Revel le soutenait déjà dans un article de L'Express publié en 1964 et intitulé "Qu'est-ce que la polémique ?"2. L'événement du Jeudi lui consacre un article de plusieurs pages en $1992^{3}$ et Le Monde diplomatique son éditorial de janvier 1995 et plusieurs articles de Manière de voir, $\mathrm{n}^{\circ} 27$ en août 1995. Ma contribution sera donc d'un autre ordre : circonscrire et définir les modalités discursives et argumentatives de la dépolitisation du lecteur et rendre compte de quelques traits spécifiques actuels de la construction médiatique du débat polémique, débat où le journaliste joue selon les cas le rôle de tiers spectateur ou celui d'acteur participant à l'affrontement.

Dernière mise au point avant d'entrer dans le vif du sujet : la dissociation proposée par Jean-François Revel entre les concepts de "polémique de ton" et "polémique de fond" sous-tend la totalité de mon argumentaire. La première est placée sous le signe de la violence verbale due à des "haines personnelles (ou 'obsessionnelles')". Tout y serait "pure affaire de ton", le "fond n'y serait pour rien"; on pourrait même "adopter" ce ton "sur commande", "comme on passe de la couleur rose sur une façade" $(132-133)$; elle est la preuve que même

1 Il s'agit d'un choix de textes qui ont été publiés de 1990 à 2003 dans des quotidiens, des hebdomadaires et des mensuels et qui ont une polémique ou le discours polémique pour objet. Les quotidiens sont Le Monde, Libération et Le Figaro ; les hebdomadaires : Le Nouvel Observateur, Le Point, L'événement du jeudi, L'Express; les mensuels : Le Monde diplomatique, Le Magazine Littéraire, Lire.

2 Cet article a été publié dans J.-F. REvel, Contrecensures, Paris, J.-J. Pauvert, 1966, pp. 132-137.

3 Cf. "Les risques de la pensée unique", 13-19.02, pp. 6-9.

4 On aura reconnu ici une thèse soutenue par plusieurs critiques de l'écriture de presse selon laquelle la pratique du devoir d'irrespect peut être parodiée à des fins manipulatoires. Il ne resterait que la posture de l'audace insolente et de l'exercice pugnace d'une fonction critique. L'enjeu réel serait double : bénéficier du prestige 
si "notre tête est entrée dans l'ère de la preuve", "notre cœur est resté dans l'ère de la force et du mensonge"(136). La polémique de fond est la seule véritable "littérature de combat" (133) à ne pas confondre avec le pamphlet. Elle ne repose pas sur la haine, le ressentiment ou la malveillance, mais sur "une nécessité vitale", "un sursaut de la sensibilité". La polémique de fond constitue une véritable menace "pour les notions ou pratiques reçues, et pour les autorités qui sont établies sur celles-ci"(133), il lui arrive d'être "combative" si l'adversaire s'entête à ne pas vouloir entendre ni comprendre, mais sans que cela soit automatique. Toute "pensée neuve", tout refus de la "servilité" sont "ouvertement ou implicitement, et parfois involontairement, polémiques" (135) ; le ton peut donc être polémique tout en restant "modéré", "pacifique"(134). Après avoir tenté de démontrer pourquoi et comment la "polémique de ton" mène à la dépolitisation de l'opinion publique, je présenterai le métadiscours de journalistes qui font l'apologie de la "polémique de fond" et de la résistance à la pensée unique et argumentent donc en faveur d'un retour au débat politique entendu comme espace public d'interaction et de négociation entre des prises de position divergentes.

\section{Métadiscours sur la dépolitisation : dénominations et définitions de quelques effets pervers du consensus...}

L'analyse des articles du corpus m'a permis de circonscrire quelques traits distinctifs, spécifiques de cette procédure. Le premier consiste à considérer la dépolitisation comme "le degré zéro de la politique". Cette dénomination est le titre d'un article du sociologue Michel Wieviorka, publié dans Libération'1. Il s'agit de la mobilisation de l'opinion et des dirigeants autour d'une prise de position "antiguerre" concernant le conflit avec le régime de Saddam Hussein. Le sociologue considère que "la bonne conscience qu'il y a à se dresser contre la guerre n'est pas une politique". Cette prise de posi-

du polémiste qui ose résister aux autorités, tout en confortant l'ordre établi et ses instances; bref il s'agit d'obtenir les gratifications de la révérence sans courir le risque de perdre la face. Cf. R. Koren, Les enjeux éthiques de l'écriture de presse, Paris, L'Harmattan, 1996, p. 140 et Manière de voir, $n^{\circ} 46$, "Ces débats médiatiquement corrects", pp. 92-93.

1 "Rebonds", 25.03.03. 
tion est également nommée dans la suite de l'article : "absence de politique", "déficit politique", "décomposition de la vie politique" "internationale, européenne et nationale", "nouvelle pensée unique"1 qui rassemble "pacifistes, propalestiniens, antimondialistes, antiaméricains, nationalistes français, souverainistes antieuropéens et beaucoup d'autres" "qui communient ainsi dans un étonnant front du refus". Ce "degré zéro" atteint même le camp adverse, favorable à la guerre, puisqu'il "procède d'un spectre singulièrement large d'hommes de droite (en majorité) et de gauche" et unit des individus aussi différents qu'un "cinéaste engagé" et un "leader libéral". Un consensus de ce genre ne pourrait résulter que d'une procédure qui consiste à "laisser de côté" des questions cruciales et à ignorer les "aberrations" que cela entraîne. La diabolisation des États-Unis impliquerait ainsi "la négation ou l'oubli des droits de l'homme ; ce dernier "s'accommode sans états d'âme" "des rapprochements diplomatiques avec la Russie et la Chine, pays qui méritent bien de graves reproches sur ce plan, et il semble oublieux des terribles méfaits de Saddam Hussein". Le consensus est donc lié à la dépolitisation par des liens essentiels quand il se résume à "rejeter, à soupçonner et dénoncer, et assurément pas à construire ou négocier". La "nouvelle pensée unique" met fin comme l'ancienne "aux camps bien délimités" : "tout" n'est plus "blanc ou noir", lit-on dans Libération (06.06.03), mais "gris", il n'y a plus d'affrontements. Les auteurs des articles cités ci-dessus mettent en cause des instances politiques, mais il existe aussi un courant critique qui dénonce le rôle central joué par

1 Cf. quant à l'analyse de variantes antérieures de la "pensée unique" : L'Événement du jeudi, 13-19.02.1992, "les risques de la pensée unique", "la dictature de l'air du temps ou le triomphe de l'information "météorologique": : $L$ 'ensemble des médias, écrits ou audiovisuels, développait le même discours, chacun confortant l'autre et le serpent se mordant indéfiniment la queue. En cela réside la principale nouveauté : quelque chose de terrifiant est en train de prendre corps qui ressemble furieusement à une pensée unique. Tout se passe comme si un même clan, réparti stratégiquement de gauche à droite, branché en direct sur 'l'air du temps', sociologiquement et culturellement homogène, tissait au jour le jour la nouvelle pensée dominante(...). Les grandes orientations définies par ce politburo occulte sont ensuite teintées d'un vernis conservateur ou progressiste en fonction des sensibilités de chacun". Cf., également, Manière de voir $\mathrm{n}^{\circ}$ 27, p. 6: "Englués. Dans les démocraties actuelles, de plus en plus de citoyens libres se sentent englués, poissés par une sorte de visqueuse doctrine qui, insensiblement, enveloppe tout raisonnement rebelle, l'inhibe, le trouble, le paralyse et finit par l'étouffer. Cette doctrine, c'est la 'pensée unique', la seule autorisée par une invisible et omniprésente police de l'opinion.". Cf., enfin, ibid., p. 14-16, "Un journalisme de révérence" par Serge Halimi. 
les médias eux-mêmes dans l'uniformisation de la pensée'. Manière de voir ( $\mathrm{n}^{\circ} 63$, p. 29) dénonce ainsi la visée des "grands médias de masse" qui ont pour objectif de "domestiquer, dresser, soumettre" leurs auditoires. Il s'agit de "réduire tout esprit critique, toute vélléité protestataire, tout rêve de révolte"2.

Autre aspect de la dépolitisation, également souligné par Wieviorka : l'affichage du ressentiment partagé qui tient lieu de programme politique, émotion stérile qui ne débouche pas sur un appel à la négociation et à la résolution des conflits, mais sur le refus de l'Autre. Ce qui compte, ce n'est plus le débat, mais le partage du refus, partage légitimé par un argument particulièrement fort : l'argument de la quantité ${ }^{3}$ qui permet, lit-on dans Le Monde du 25.03.03 ("Point de vue" par Robert Redeker), "de voir dans le nombre la raison". Or plus d" "échanges" ni de "concurrences" et de "dérégulation" par "un double permanent et contraire", partant "plus de politique", affirment Bonnafous et Tournier".

Le refus de toute interaction verbale avec l'adversaire diabolisé serait dû, selon Le Monde (06.03.03), à "un invraisemblable déferlement de haine". La dépolitisation résulte donc, en l'occurrence, de la transformation de l'affrontement dialogique en "inquisition d'un autre âge", "jugements assassins" (ibid.), "attaques outrancières" ( Le Figaro 06.05.03), "réquisitoire implacable", "virulente philippique" (Le Point 14.02.03). Nous sommes exactement dans le cas d'une "parole envahissante" qui refuse de "dialoguer", de la "clôture" ou

1 Cf., à ce sujet M. DOURy, Le débat immobile, Paris, Kimé, 1997 et R. Koren, op. cit., pp. 259-267 ainsi que R. KOREN, "Quand informer, c'est dire le blanc et le noir: quelques effets pervers de l'oscillation binaire...", in P. BoGAARDS, J. ROORYKC et P. J. SMITH (dir.), Quitte ou Double Sens, Amsterdam, Rodopi, 2001, pp. 177-200.

2 Le Monde diplomatique dénonçait déjà cette forme d'élaboration de la "pensée unique", dans Manière de voir $n^{\circ} 14$, p. 31, en février 1992 et dans le $n^{\circ} 27$, p. 7 , en août 1995, en ces termes: "Les communications de masse, les industries de l'information et du spectacle imprègnent de leur idéologie molle l'ensemble des activités des citoyens, imposant, au-delà de la souveraineté de l'État, une véritable uniformisation de la pensée" (1992) "un consensus idéologique écrasant, imposé par les médias, par les sondages et par la publicité, grâce à la manipulation des signes et des symboles et aux nouvelles armes du contrôle des esprits" (1995).

3 Cf., à propos des lieux de la quantité ou de la qualité, C. PERELMan et L. OlBRECHTS-TyTECA, Le traité de l'argumentation. La Nouvelle Rhétorique, Bruxelles, Éd. de l'Université de Bruxelles, 4e éd., 1970, pp. 115-125.

4 S. BOnNafous, M. TOURnIER, "Analyse du discours, lexicométrie, communication et politique", in D. MAINGUENEAU (dir.), "Les analyses du discours en France", Langages, $\mathrm{n}^{\circ} 127$, mars 1995 , p. 68. 
"dialogue outrepassé" qui entraîne "la perte de l'espace public" et réduit la parole de l'adversaire à des "bruits incompris, voire au silence" (Bonnafous et Tournier). La rhétorique pacifiste hostile à la guerre contre l'Irak constitue un exemple type aux yeux de Robert Redeker, auteur d'un article ("Point de vue") publié dans Le Monde du 26.03.03. Ce qui mérite, tout d'abord, de retenir l'attention, c'est le fait paradoxal que cette rhétorique "pacifiste" est "peu pacifique dans la virulence agressive de ses énoncés". Ce "néopacifisme planétaire" tient un discours idéologique "partisan", "violemment agressif" ; il est, par sa "violence" et son "hostilité", "un autre discours de la guerre". La "dépolitisation" est un des effets du "refus ressentimental".

\section{Justifications de la thèse de l" "infamie" de la polémique de ton}

Si telle est l'image de la polémique, image dominante qui occulte ou marginalise son double politique : la polémique de fond, il n'est donc pas étonnant qu'on la trouve suspecte et même "infâme"l et que l'on rencontre fréquemment des énoncés où l'on se défend d'avoir des intentions polémiques et où l'on entoure sa prise de position de précautions verbales aussi explicites que possible. Il suffit en effet, pour discréditer un point de vue, de le traiter de "polémique" 2 . On se

1 Cf. C. Kerbrat-Orecchioni, "La polémique et ses définitions", in Le disdcours polémique, Lyon, PUL, "Linguistique et sémiologie", 7, 1980, p. 40 : "On l'aime [la polémique] et elle fascine, perversement, mais on affirme ne pas l'aimer : la polémique est infamante (Hugo, cité par Robert : 'L'abbé Grégoire, ancien évêque, ancien conventionnel, ancien sénateur, était passé dans la polémique royaliste à l'état d'infâme Grégoire') autant pour celui qui l'exerce que pour celui contre lequel elle s'exerce".

2 "Tout au long de l'année 2000, écrit Marc-Antoine Coppo, cité par P. PEAN, $\mathrm{Ph}$. COHEN, (La face cachée du Monde. Du contre-pouvoir aux abus de pouvoir, Paris, Mille et Une Nuits, 2003, p. 533), alors que se multiplient dans la presse étrangère les information gênantes, voire contredisant carrément les thèses du quotidien, Le Monde s'applique méthodiquement à transformer ces révélations en simples 'polémiques". Cf., également, cet extrait de Libération (21.08.03) : "En vacances en Haute-Savoie pendant la crise, il [le Premier ministre] a visiblement eu du mal à évaluer la situation, qualifiant le 12 août, les critiques de la gauche de 'polémiques partisanes'". Il apparaît clairement, dans ce commentaire d'un ouvrage de P.-A. Taguieff, que "polémique" est un terme axiologique négatif : "le dernier livre de Taguieff a été violemment attaqué : il part pourtant d'une intention bonne, quoique polémique, dirigée à la fois contre les belles âmes et contre le sens 
sert de ce terme pour disqualifier l'opposant dissident qui conteste l'opinion doxique dominante : le proposant conforme à l'orthodoxie serait l'unique dépositaire de la Vérité. Du discrédit, dû à la violence verbale, à la dépolitisation, il n'y a qu'un pas.

Le métadiscours anonyme sur ce type de guerre des mots en dénonce les paradoxes : mode d'interaction verbale essentiellement dialogique, elle est cependant le plus souvent un dialogue de sourds où règne l'interincomprehension ${ }^{1}$; le but de l'affrontement est de vaincre, mais rien ne garantit que la parole de l'Autre ne finisse pas par avoir raison de l'Un ; les gains ne sont pas nécessairement nuls, mais ce ne sont pas toujours ceux que l'on pensait ou désirait obtenir : à défaut de réussir à déstabiliser l'adversaire, on se trouve confronté à ses propres prises de position et la confrontation n'est pas toujours flatteuse ni rassurante. La polémique de ton est sectaire et spectaculaire, il n'est donc pas étonnant que ceux auxquels elle s'attaque se sentent menacés et cherchent à s'y soustraire.

Ses détracteurs lui reprochent essentiellement de recourir à des formes de violence ${ }^{2}$ incompatibles avec le bon fonctionnement de la vie sociale, de transgresser les règles de l'éthique de l'action et de déposséder l'auditoire de sa liberté de penser. Les auteurs du corpus dénoncent essentiellement deux formes de violence argumentative : la violence symbolique des "attaques ad hominem" qui "fixent le ton et le registre" (Manière de voir, $n^{\circ} 63, \mathrm{p} .64$ ) et le caractère incontrôlable d'un affrontement qui implique tous les dangers de l'engrenage. L'attaque consiste à neutraliser les velléités de débat pertinent en publiant, déclare Régis Debray, "des injures ad hominem" qui passent "sous silence des questions de fond "(ibid., p. 67). Ce qui semble condamnable, c'est la "brutalisation", la "montée des incivilités", la "dégradation des échanges"3. Quant au danger de l'engrenage incontrôlable, voici comment il est perçu, défini et affronté par Le Monde dans trois articles consacrés à la polémique contre l'ouvrage de Péan

commun" Le Magazine littéraire, ${ }^{\circ}$ 333, juin 1995, p. 91. Cf. enfin J.-F.. FoGEL, L. Greilsamer et D. RoynetTe, Le style du Monde, (Paris, Le Monde, 2002, p. 7) où "polémique" est mis sur le même plan que "sarcasme" et "attaques personnelles".

1 Cf., à propos de l'“interincompréhension": D. Maingueneau, Sémantique de la polémique, Lausanne, L'Age d'Homme, 1983, p. 23.

2 Il aurait été utile et pertinent de rendre compte ici de la mise en mots médiatique de la violence verbale des insultes échangées par les acteurs impliqués dans les polémiques, mais j'y renonce, faute de place.

3 "Règlements de comptes chez les intellectuels", Lire, févr. 2003, p. 30. 
et Cohen : "chaque réponse, dans ce jeu confusionnel et pervers, entraîne alors une nouvelle question" (06.03.03), "Par quel bout prendre la machine infernale de Péan et Cohen ? A peine réplique-ton sur un point qu'on est sommé de s'expliquer sur trois autres, de nature tout à fait différente, auxquels il est parfois impossible de répondre." (08.03.03). "Pour répondre au livre, la direction du journal a choisi la stratégie suivante : (..) - ne pas accepter d'interviews ou de débats sur les ondes" (01.03.03).

Autre argument de poids dans la procédure de délégitimation de la polémique : les transgressions de l'éthique du discours liées à l'axiome la fin justifie les moyens. Première illustration : l'ancien militant de la gauche ou de l'extrême-gauche, "en particulier dans les médias", qui s'est rallié, affirme J.-F. Kahn, au "pancapitalisme le plus implacable et le plus oppressif" serait prêt à tout faire pour maintenir son image de marque gauchiste...Tout, c'est-à-dire : "exploitation forcenée des thèmes qui, sans en rien remettre en cause l'ordre social, les 'marque' à gauche", mais aussi, si cela devait devenir "un thème fédérateur qui permette de se positionner 'progressiste"" aller jusqu'à "diaboliser Jean Moulin" ou "réhabiliter le général Vlassov" (L'Evénement du Jeudi, "Comment avec l'abbé Pierre, on sert la soupe à Le Pen et Faurisson" 02-08.05.1996). Seconde illustration: les fins idéologiques de la "bonne presse" "centre gauche" masquées par une rhétorique pseudo-objective "pharisienne" qui consiste à "observer, affirme R. Debray, les rituels de l'objectivité en les vidant de leur sens : les signes de la vertu, sans la vertu. La messe sans la foi" (Manière de voir, $\mathrm{n}^{\circ} 63$, p. 66). Les jeux du paraître neutre garantissent la légitimation de l'opinion publique ${ }^{1}$. Troisième illustration : dans une chronique du Monde (28.02.03) où il répond à une attaque dirigée contre lui par un confrère de Libération (Pierre Marcelle), qui accuse le journal de cette "abominable duplicité" : "Le Monde imprimerie imprime ce que Le Monde-quotidien dénonce", Pierre Georges achève l'article par la remarque suivante, sans rapport logique direct avec la contradiction qui oppose l'imprimerie au "Monde-quotidien": "Pierre Marcelle lit les bons auteurs, 'Péan (et Cohen)' je le cite. Cela fait deux fois en deux chroniques qu'il met le nom de Cohen entre parenthèses. Seraient-ce des parenthèses symptomatiques, docteur?". La fin :

1 Cf., à propos des effets d'objectivité à l'œuvre dans la rhétorique de l'écriture de presse, R. KOREN, Les enjeux éthiques de l'écriture de presse, op. cit., pp. 53-107. 
détourner l'attention des "contradictions" du Monde, en banaliser la gravité -justifie le "moyen" : reléguer dans l'implicite un sousentendu qui accuse Pierre Marcel de discrimination antisémite.

\section{De quelques stratégies discursives de la dépolitisation}

Il existe, indépendamment de l'information spectacle qui augmente la visibilité de la polémique de ton, des stratégies moins visibles destinées à détourner l'attention du lecteur des problèmes de fond en déplaçant les feux de la rampe vers des objets de débat de moindre importance. Je me propose d'analyser à présent quelquesunes d'entre elles. De la désinformation qui en résulte à la dépolitisation, il n'y a qu'un pas : peut-on contester ce qu'on ignore, ce qui est passé sous silence ? La procédure est définie de façon particulièrement significative par Régis Debray dans un paragraphe d' "une machine de guerre" intitulé "L'effet paravent", dénomination empruntée au rédacteur en chef du Monde diplomatique, Ignacio Ramonet. Il s'agit de 1" "escamotage du fond par la forme", de l'"occultation du majeur par le mineur" ; l'énonciateur "focalise sur un point particulier, qui, d'illustratif qu'il était, devient emblématique" (Manière de voir, $\left.\mathrm{n}^{\circ} 63, \mathrm{p} .65\right)$ et fait comme s'il était le point de litige fondamental.

Je me contente, faute de place, de classer et de définir les divers "effets paravents". Le premier type de recadrage de l'objet du débat consiste à "focaliser sur un point" techniquel qui permet d'escamoter un différend politique ou déontologique concernant les "véritables" enjeux de l'information. La polémique autour du thème évoqué cidessus : "Le Monde imprimerie imprime ce que Le Monde-quotidien dénonce" ( Le Monde 28.02.03, chronique de Pierre Georges) substi-

1 Opposer le point de vue technique à l'engagement politique n'est pas polémique en soi comme en témoigne cette remarque relevée dans un article du Figaro: "la nouvelle génération" engagée dans l'action humanitaire y est présentée comme plus "technicienne", entendu comme délibérément "dépolitisée". Ce qui résulte ici d'un choix explicite ne saurait être confondu avec la stratégie dissimulatrice définie cidessus. 
tue des considérations économiques purement techniques ${ }^{1}$ aux causes éthiques invoquées par l'adversaire.

Autre type de masquage : la scénographie du "professionnel" qui "décoche" des "foudres 'strictement techniques", (Debray, Manière de voir, $\mathrm{n}^{\circ} 63$, p. 66). L'objet du débat n'est pas alors les dissensions politiques, mais la transgression répétée de règles méthodologiques. La polémique sur des questions de fond est remplacée par des critiques de grand professionnel sur les erreurs déontologiques commises par des amateurs incompétents. Le Monde est pour l'engagement militaire de la France dans le conflit du Kosovo ; Debray est contre et le journal attaque son argumentaire en ces termes : "une telle accumulation d'erreurs élémentaires -on parle ici, d'un point de vue strictement professionnel, du minimum exigible d'un élève de première année en école de journalisme sur la vérification et le recoupement des informations, la multiplicité des sources et le refus des impressions" (Pierre Georges cité par Debray, Manière de voir, $\mathrm{n}^{\circ} 63$, p. 67). La procédure de dépolitisation de l'information et de l'auditoire peut également consister dans la substitution du pathos au logos : c'est alors la "sentimentalité" qui tient lieu de "civisme"2. Les passions, l'émotion jouent un rôle crucial en politique ; sans elles, pas d'identification ni de passage à l'action. Qui dit pathos ne dit pas renoncement à toute logique : on peut être parfaitement capable de rationaliser ses émotions et ses jugements de valeur, entendu comme les argumenter, les justifier ${ }^{3}$. Le problème est donc ailleurs, dans la stratégie qui consiste à renoncer à la discussion de programmes politiques et à miser essentiellement sur les sentiments ${ }^{4}$. L'analyse des

1 Cette qualification n'est pas péjorative en soi, elle le devient quand elle sert à occulter les vrais débats comme l'indique ce passage d'un article de Libération : "Ia dimension purement comptable qui pèse sur cette réforme achève de faire fuir les 'penseurs "au profit des 'experts"". "Relevant d'un 'choix de société, la réforme s'est réduite à un débat technique, très technique" (06.06.03).

2 Cf. Ph. MEYer, "Retrouver le goût de la polémique et des vrais débats", Le Bulletin Gallimard, no 413, mai-juin 1996, p. 16 : "Conformisme et pensée 'correcte' ne cessent de gagner du terrain. La dérision tient lieu d'humour et la sentimentalité de civisme...".

3 Cf., au sujet de "la rationalité axiologique", R. Boudon, "Sens et raisons : théorie de l'argumentation et sciences humaines", Hermès, n 16, 1995, pp. 29-41.

4 La démarche de la presse écrite est comparable en l'occurrence à celle de certains hommes politiques: "Le comble de la mystification est atteint, affirme François Brune, lorsque nos dirigeants font état publiquement de leur for intérieur : compassion formelle, humanisme au grand cour (l'évocation de la crise), sentiments désintéressés, convictions profondes, credo prophétique (le chant des 
causes du combat de Péan et Cohen met l'accent dans les colonnes du Monde sur "un invraisemblable sentiment de haine" (Jean Colombani, le 06.03.03). "La haine est un sentiment mystérieux", précise Dominique Dhombres le 07.03.03, ce n'est pas “au fond ceci ou cela que nous reprochent Pierre Péan et Philippe Cohen. C'est tout simplement d'exister". Or si le sentiment de "haine" est la cause de tous ces maux, "il n'y a pas grand-chose à répondre"... Discuter avec des êtres irrationnels est absolument inutile. La victimisation du Monde et la diabolisation de ses adversaires sont ici à l'origine de la dépolitisation du débat.

L'analyse de quelques articles consacrés à la commémoration louangeuse d'hommes célèbres impliqués dans des polémiques notoires révèle l'existence du type de dépolitisation suivant : la récupération du dissident défunt par les instances du pouvoir, qu'il a combattues toute sa vie et qui remportent ainsi, après sa mort, ironie du destin, une victoire impossible auparavant. Jacques Julliard affirme ainsi après la mort de Pierre Bourdieu que "L'unanimité de l'hommage posthume traduit l'échec éclatant de Pierre Bourdieu" : "la preuve est ainsi faite que la démocratie consensuelle est un enzyme capable de digérer la critique la plus radicale"; même "Le Nouvel Obs', sa bête noire, le symbole éclairé de la bourgeoisie moderniste, n'a cessé de lui rendre hommage" (Le Nouvel Observateur, 31.01-06.02.02).

Dernier type de stratégie de dépolitisation relevé dans mon corpus : l'apologie des plaisirs apolitiques de "l'illusion groupale", définie en ces termes, par Max Dorra, dans Le Monde diplomatique de juin 1996 ("La traversée des apparences") : "Les idées reçues peuvent procurer un plaisir. Celui de se fondre incognito dans la masse. Le plaisir mimétique de l' illusion groupale"". Serge Halimi abonde dans son sens dans le cadre du portrait ironique d'une consœur "qui connaît l'usage du terrible contre-pouvoir des médias : elle fut successivement chef du service politique au Point, PDG de Radio France, présidente de la Haute Autorité de la communication audiovisuelle et directrice de l'information de TF1." Il serait donc légitime de penser, "usage du contre-pouvoir" oblige, que "ses projets bruissent d'ambitions subversives", or il n'en est rien, bien au contraire. Ce qu'elle prône, c'est une nouvelle émission, "Alors heureux", "magazine d'actualité posi-

promesses). Trop de responsables préfêrent le langage qui s'apitoie au discours qui analyse" Manière de voir, $\mathrm{n}^{\circ} 27, \mathrm{p} .24$. 
tive", défini en ces termes, cités par Halimi : "Il faut suivre l'air du temps, nous ne sommes plus dans une France de polémiques : deux millions de personnes sont allées voir les voiliers sur l'Atlantique, tout le monde s'est mobilisé pour l'éclipse. Les gens ne demandent qu'à se rassembler" (Manière de voir, $\mathrm{n}^{\circ} 63$, p. 53). Cette déclaration présente le conflit politique, l'affrontement de positions divergentes comme anachronique. L'auteur de ces lignes fait l'apologie d'un espace public dont les thèmes fédérateurs : "les voiliers", "l'éclipse", offrent les plaisirs de "l'illusion groupale" et libèrent du joug de l'engagement politique. Comment s'étonner dès lors que le politique et la polémique de fond se fassent rares...

\section{Quelle polémique pour quel espace public?}

Je passe à présent à l'analyse de deux exemples types de la construction médiatique de l'espace polémique et de la dépolitisation du débat. Le premier cas concerne une polémique dont les protagonistes sont R. Debray et B.H. Levy. Debray publie dans Le Monde du 13.05.1999 un article intitulé "Lettre d'un voyageur au président de la République"; la lettre est donc adressée à un homme politique qui représente le pouvoir exécutif et non pas à Levy ni au journal $L e$ Monde. C'est donc ce dernier qui prend l'initiative de construire l'espace du débat de fond suscité par les prises de position de Debray et de distribuer les rôles comme il l'entend. Les opposants choisis par le journal (B.H.L. n'est pas le seul opposant sollicité par la rédaction) ne sont pas les porte-parole du président, mais des intellectuels notoires ; il y a donc une procédure de dépolitisation déclenchée par le choix de ceux qui joueront le rôle de l'opposant. La procédure est partielle : les protagonistes s'affrontent sur des questions de fond concernant l'engagement militaire de la France, engagement justifié par le respect de valeurs républicaines essentielles; le politique est donc sauf sur ce point. Ce qui ne l'est pas, par contre, c'est le droit de choisir son coénonciateur et le cadre de l'énonciation. "Je ne m'adressais nullement à B.H. L., souligne Debray, (...). Ce décadrage ne pouvait que folkloriser (éternelle parisianité), personnaliser (...), idéologiser (...) un débat qui se voulait sérieux, pragmatique et surtout contemporain. Il le faisait verser côté aboyeurs (B.H.L. pratique en effet abondamment la polémique de ton), non côté déci- 
deurs, en y intéressant les salons, non les bureaux" (Manière de voir, $n^{\circ} 63$, p. 64) ${ }^{1}$.

Qu'en est-il lorsque Le Monde est lui-même la cible de polémistes ? Je n'analyserai pas la totalité de l'affrontement qui l'oppose à Péan et Cohen : ce "combat" a les qualités du cas d'école et mérite une étude en soi ${ }^{2}$. Je voudrais juste mettre l'accent sur l'ethos du Monde tel qu'il apparaît au début de la polémique et sur les implications de cette image pour la construction de l'espace du débat. "Attaquée, diffamée, insultée, la rédaction du Monde est blessée", "c'est l'ensemble de la collectivité du Monde (...) qui est livré à l'opprobre, à la vindicte de l'opinion, de ses lecteurs, de ses actionnaires et de ses alliés", lit-on le 28.02.03 dans les colonnes du journal. Ce discours est bien, comme le remarque un journaliste de Lire (avril 2003, p. 22) celui de "vestales qui gardent le temple sacré" et prennent un air "effarouché", mais aussi, simultanément, le discours grandiloquent de la Victime menacée dans son existence même. Si les arguments de Péan et Cohen sont essentiellement présentés comme inspirés par une haine inexplicable et par la violence verbale du pamphlet partisan et meurtrier, il peut donc paraître légitime de "ne

1 J'ai pu observer une stratégie de cadrage similaire dans un tout autre contexte : la présentation de la "polémique" entre l'Autorité palestinienne et l'État d'Israël lors de l'affaire du Karine A, bateau intercepté par l'armée israélienne et qui transportait une très importante quantité d'armes. Israël a tenté en vain de persuader l'opinion publique internationale que l'Autorité était le commanditaire de ces armes ; l'Autorité a nié cette accusation opiniâtrement. L'intervention du Monde (08.01.02) consiste, entre autres, à donner la parole à Arik Sharon dans la rubrique "Verbatim" ("Ariel Sharon: 'Arafat est un menteur"') et à lui opposer, non pas des déclarations de Yasser Arafat, mais un communiqué de la Lloyd"s List, "publication qui fait autorité en matière de transport maritime" et qui "conteste la version israélienne de l'arraisonnement du Karine $A$ "; suivent une série d'hypothèses de la publication londonienne sur les éventuels commanditaires, qui mettent en doute systématiquement le point de vue israélien. Cet agencement de l'affrontement polémique surprend : le traitement de la "controverse" est en effet défini en ces termes dans Le Style du Monde : "En cas de controverse, il est nécessaire de faire connaître les diverses versions d'un événement et les divers points de vue de ses acteurs" (p. 7) ; Sharon est un "acteur", Arafat aussi, mais La Lloyd's List est un tiers. Le Monde ne crée donc pas ici les conditions de possibilité d'un espace de débat politique; il orchestre un "non-débat" qui a uniquement les apparences du dialogue et qui constitue de ce fait un obstacle au politique. Il aurait dû jouer, conformément à son propre code déontologique, le rôle du tiers médiateur qui exerce une fonction critique équitable or il attribue le rôle de l'opposant à un acteur qui n'est pas l'opposant naturel. Il y aurait aussi beaucoup à dire sur cet étrange paternalisme qui consiste à confier la défense de l'“accusé" à un tiers et pas à l'accusé lui-même...

2 Il est l'objet ici même d'un travail de recherche : cf. l'article de Raphaël Micheli. 
pas accepter d'interviews ou de débats sur les ondes" débat immédiatement accepté et mis en vedette est celui revendiqué par la Société des rédacteurs du Monde, débat entre membres de la même collectivité, que légitime la "charge", mais qui ne saurait tenir lieu d'affrontement avec l'adversaire (cf. Le Monde, 28.02.03). Le refus initial de débattre finit par être remis en question; Daniel Schneidermann ("Parole contre parole", "enfin!", 07.03.03) ${ }^{2}$ se félicite qu'il y ait enfin "plus de quinze jours après le début du séisme" "des réponses des accusés, des réponses en longueur que l'on peut lire, écouter, relire, comparer, confronter aux accusations du livre"; mais les interventions de Solé et de Schneidermann ressemblent étrangement à ce que Serge Halimi nomme dans Manière de voir ( $\left.\mathrm{n}^{\circ} 46\right)$ : "un ilôt-alibi dans un océan de pensée conforme".

\section{Apologie de la polémique de fond}

L'argumentation qui consiste à promouvoir la pratique du débat politique combatif et la résistance aux pressions de la pensée unique est certes minoritaire, mais il existe "un discours contre"3 et je me propose, à présent, de reconstituer les définitions métadiscursives de la polémique de fond qui le caractérisent. Je commencerai, toutefois, par rappeler la définition du "texte politique" et des rapports "entre politique et phénomènes langagiers", proposée par Bonnafous et Tournier ${ }^{4}$; cette définition devrait augmenter la visibilité des traits spécifiques du métadiscours à l'œuvre dans l'écriture de presse

1 Cette stratégie est la troisième de celles énumérées par le médiateur Robert Solé dans "Face-à-face" (01.03.03), les quatre autres sont les suivantes: "dénoncer le procès 'idéologique' fait aux trois dirigeants du Monde", "souligner qu'au-delà de leur personnes, c'est le journal qu'on cherche à déstabiliser", "s'assurer avant tout de la solidarité des différentes catégories de personnels de l'entreprise", "engager des poursuites judiciaires".

2 Cf., également, l'article du médiateur, Robert Solé ("Après la tourmente", 08.03.03).

3 J'emprunte cette dénomination à C. Plantin, "Analyse et critique du discours argumentatif", in R. KOREN et R. AMOSsY (dir.), Après Perelman : quelles politiques pour les nouvelles r'hétoriques?, Paris, L'Harmattan, 2002, pp. 230. Il y affirme, entre autres, que si le chercheur en sciences du langage n'a pas à évaluer les prises de position des protagonistes d'une controverse en termes éthiques, il existe néanmoins un type d'engagement scientifique légitime : opposer au discours pour, un "discours contre" sélectionné par le chercheur.

4 S. Bonaffous et M. TOURnier, op. cit., pp. 67-68. 
actuelle. Si la politique est "un pouvoir dans l'organisation du social", elle est nécessairement liée à un "système" de "signes par où passent les reconnaissances et représentations, grâce auxquelles le social s'érige une cohérence", affirment Bonnafous et Tournier ; mais "il n'y a pas non plus organisation sans son double permanent et contraire, l'ensemble des forces de dispersion, de dérégulation, de mise en cause des pouvoirs ou des code organisateurs. Cette mise en cause passe, elle aussi, par les signes, souvent les mêmes signes, porteurs à leur envers de négativité". La politique serait donc, dans cette perspective, "une lutte pour la stabilisation ou la déstabilisation langagières, où n'existent que de faux armistices sur des valeurs de langue"'. Le fait que les oppositions "stabilisation \déstabilisation", "cohérence \dérégulation" soient présentées comme inhérentes à l'organisation politique de la vie sociale augmente la visibilité de la dépolitisation qui caractérise l'opinion publique actuelle. Ce qui devrait aller de soi et être perçu comme une dynamique saine, souhaitable et incontournable, doit aujourd'hui être justifié et affronter les obstacles érigés par le camp de 1"“organisation". La divergence n'est pas perçue comme un constituant bénéfique nécessaire, mais comme un mal qu'il faut juguler. On prône certes le débat et les rubriques "débats" sont de plus en plus fréquentes dans la presse écrite comme dans les médias audiovisuels : il est "médiatiquement" et démocratiquement "correct" de tenir ce genre de discours. Ce qui orchestre cependant l'espace public, ce sont les non-dits d'une pensée unique toute-puissante qui délégitime implicitement ce qu'elle semble approuver explicitement. C'est "en réinventant des débats et des conflits sociaux et culturels que notre société pourra se doter des repères autorisant la recomposition d'un espace politique national, où les idées de gauche et de droite voudront vraiment dire quelque chose", conclut Wieviorka dans "Le degré zéro de la politique"(Libération, 25.03.03).

Les voix médiatiques qui défendent la polémique de fond exigent que l'information allie "humeur et réflexion", "raison" et "résistance"2, diffusion d'un savoir civil et "ton offensif" ou "rage" "qui donne envie de se battre pour une société (...) plus humaine, plus

I Ibid., p. 69.

2 Cf. respectivement, Le Nouvel Observateur, "La passion selon Julliard", 2127.03.1991 et Le Bulletin Gallimard, ${ }^{\circ}$ 413, mai-juin 1996, "retrouver le goût de la polémique". 
sociale"l. Elles retrouvent ce faisant ce qui a toujours été considéré comme une opposition fondatrice de la polémique : la concomitance et l'interaction de "la techné, de la maîtrise" et de "la spontanéité, de la sincérité, de l'engagement passionnel", du "didactisme et de l'emportement"' Le médiateur du Monde, Robert Solé, problématise dans sa chronique du 22.02.03 intitulée "Tous en cœur" le débat pour ou contre la guerre contre l'Irak et l'unanimité du "consensus affiché". Il y déclare que le journal a clos la rubrique qui servait de cadre à la controverse, le 21 février, environ trois semaines après son ouverture, faute de "va-t-en guerre" désireux de contester l'unanimité des pacifistes. Solé déclare enfin, avant de passer à un autre débat sur le délit "d'outrage au drapeau tricolore et l'hymne national" dans le cadre de matchs de football, que "les Français en général et les lecteurs du Monde en particulier sont très majoritairement opposés à la guerre préventive". Sa prise de position -le médiateur est autorisé à réagir subjectivement ${ }^{3}$ - consiste essentiellement dans le ton de dérision qui accompagne la description des réactions des lecteurs, dérision qui appartient au registre de la polémique de ton (gouaille du niveau de langue intentionnellement familier) et dans l'hypothèse que la guerre pourrait être "au service de l'unité nationale". La distance entre ce mode d'approche de la pensée unique et l'argumentaire de l'auteur d'un autre point de vue critique sur ce même consensus : "Les néopacifistes en guerre... contre la paix" (Le Monde 25.03.03) est considérable. Il s'agit certes de deux genres de textes différents : l'un donne la parole au médiateur, l'autre à un invité, Robert Redeker, qui n'est pas journaliste, mais professeur de lycée et "membre du comité de rédaction de la revue Les Temps modernes". Le mérite d'avoir donné la parole à un auteur aussi compétent et "offensif"4 revient au Monde, mais ne change rien au fait que le médiateur soit resté silencieux quant aux dangers du "consensus affiché" : il le tourne en déri-

1 Cf. la définition de l'esprit "Charlie Hebdo" proposée par Le Nouvel Observateur, 12-18. 09.1996.

2 Cf. Kerbrat-Orecchionii, Le discours polémique, Lyon, Presses universitaires de Lyon, 1980 , pp. 20-21.

3 Cf. Le style du Monde, op.cit., p. 71 : "La chronique publiée par le médiateur a plusieurs fonctions : (...) réfléchir sur le contenu, la forme et la manière de faire le journal ; donner un avis personnel (...). Le médiateur est indépendant de la rédaction".

4 Cf . quant à l'emploi de cette épithète, Wieviorka, "Le degré zéro de la politique" : "demander à la droite atlantiste d'avoir le courage de se dévoiler, et à la gauche de faire des propositions offensives et réalistes (...) pour animer la vie sociale. Nous avons besoin de plus de politique". 
sion, mais ne se prononce pas sur les tenants et aboutissants d'un tel conformisme. Il contribue également, par ailleurs, à la dépolitisation de l'auditoire en mettant sur le même plan les débats polémiques autour de la guerre et le rituel des matchs de football... Le lecteur pourra-t-il évaluer les enjeux du consensus pacifiste en connaissance de cause et aura-t-il encore envie de prendre position ? L'analyse de Redeker formule des hypothèses déstabilisatrices quant à l' "occultation" des "racines" et de "l'histoire" du "pacifisme contemporain", mais aussi quant à son "objectif secret" : "faire oublier un événement tout aussi important que la victoire des Américains sur le nazisme hitlérien, jamais signalé. L'événement refoulé, objet du tabou mémoriel, dura plusieurs décennies: l'Amérique a protégé l'Europe occidentale du communisme". Redeker insiste sur quelques paradoxes propices à la réflexion : la rhétorique du néopacifisme est, contrairement à toute attente, "virulente" et "violemment agressive".

Qu'est-ce que la polémique de fond, dans ces conditions ? La résistance, le courage de "nager contre le courant", d'"être extrêmement minoritaire" (Nicolas Weill, propos de Glucksman, Bruckner et Goupil, Le Monde 19.03.03) et d'oser s'opposer, comme l'affirme Debray (Manière de voir, $\mathrm{n}^{\circ} 63$, p. 68) "au visage actuel du fanatisme". Celui-ci se reconnaît aux actes de langage suivants: "terroriser par l'adjectif, sans démonstration", "ne pas établir, asséner", "déconsidérer" le "porteur d'une thèse" contestataire plutôt que d'en discuter avec lui, afficher les apparences de la "Vérité infuse", arborer un ton "outrancier, théâtral, fulminatoire", "se battre publiquement à vingt contre un", utiliser "le nombre, l'écran, les caricaturistes et la grande presse" pour tenir la cible "en respect", bref "usurper la place de l'esprit libre".

\section{Quelques mots de conclusion...}

On pourrait être tenté de croire, à force de lire les argumentaires qui dénoncent la pensée unique consensuelle et qui louent la polémique de fond, que la première est toujours inquiétante et la seconde toujours bénéfique ; mais il me faut insister, au moment de conclure, sur la nécessité d'éviter l'écueil des prises de position dichotomiques qui pourraient nous empêcher de penser les situations complexes etlou exceptionnelles. Le rassemblement ne constitue pas une menace en 
soi, bien au contraire, mais l'enjeu du rassemblement peut déclencher des effets pervers problématiques.

Dernières remarques avant d'illustrer ces affirmations : les pôles de la "stabilisation" et de la "déstabilisation" sont des pôles antithéthiques, mais interdépendants. Pas de vie politique possible sans "gestion de la vie collective ni régulation", affirment Bonnafous et Tournier. La "diabolisation" du consensus risquerait de faire oublier que si l'unité de façade ${ }^{1}$, la posture conformiste dictée par le refus de l'Autre sont problématiques, la solidarité entre membres d'une même collectivité ou entre adversaires politiques qui partagent des valeurs communes revêt une importance cruciale en temps de guerre ou de conflit de politique intérieure. L'"organisation" et la "dérégulation" sont aussi importantes l'une que l'autre... L'écrivain et essayiste Peter Schneider dénonce dans "Les débats de l'Obs", l'unanimité "angoissante" contre la guerre, en Allemagne, mais il argumente, simultanément, en faveur d'une proposition de Jürgen Habermas et Jacques Derrida qui prône un regain de l'union européenne : les "pays européens pionniers, qui il y a cinquante ans ont donné l'impulsion à l'union économique, devraient maintenant s'assembler pour former une union politique et créer ainsi un modèle pour le reste de l'Europe". Le consensus peut également s'avérer bénéfique, par ailleurs, s'il représente la récompense d' "un combat contre la discrimination" : le fait que la droite se soit sentie obligée de "se joindre au cortège" dénommé "marche des fiertés lesbienne, gay, bi et trans" est présenté dans un éditorial de Libération (28.06.03) comme une victoire. Les connotations négatives de la dépolitisation due à l'uniformisation de la droite et de la gauche sont inversées ici par la fin éthique du combat contre l'exclusion.

L'apologie de la polémique de fond doit également être problématisée. J.-F. Kahn soutient ainsi, dans "Comment, avec l'abbé Pierre, on sert la soupe à Le Pen et Faurisson", que les médias "s'échinent" parfois à promouvoir ce qu'ils prétendent combattre et redouter par-dessus tout"2. Autrement dit, affronter, "évoquer",

1 Cf., à ce sujet, Le Monde du 01.03.03, "Face-à-face", où le médiateur déclare : "la rédaction est suffisamment unie pour ne pas vouloir apparaître uniforme, comme le lui reprochent ses détracteurs".

2 Cette thèse est également pertinente dans le cas du terrorisme où les médias donnent aux idéologues des mouvements terroristes interviewés ou cités une scène d'énonciation qui augmente l'impact de leurs dires et les institutionnalise là où il faudrait surtout les criminaliser. 
"décortiquer", "soupeser", "discuter les thèses" de l'adversaire, thèses que l'on proclame "taboues", peut aboutir, par exemple, dans le cas d'un Le Pen, d'un Faurisson ou d'un Garaudy à "s'en faire inconsciemment les complices", à donner à "un libelle" une "dimension considérable" : "il devient le centre d'un débat, les thèses qu'il défend sont portées à la connaissance du plus grand nombre, il fait la 'une' de tous les grands journaux. Publicité inespérée. Consécration inouie" (L'événement du Jeudi, 02-08.05.1996). La polémique de fond est détournée ici de son but combatif par des causes liées à la nature du langage (tout ce dont on parle est aussitôt valorisé du fait même d'avoir été sélectionné et mis en mots) et aux tentations de l'information spectacle (un scandale, une affaire sont une aubaine pour les médias).

Le Monde vient de publier en juilletlaoût 2003 une série de douze portraits intitulée "Seuls contre tous". Les "personnages célèbres" sélectionnés sont par ordre chronologique : "Diogène le cynique" (19.07.03), "Rabelais l'insolent" (21.07.03), "Galilée le présomptueux" (22.07.03), "Spinoza le maudit" (23.07.03), "Olympe (de Gouges) l'imprécatrice" (24.07.03), "Schopenhauer l'incompris" (25.07.03), "Darwin l'opiniâtre" (26.07.03), "Courbet le peintre" (28.07.03), "Louise Michel la combattante" (29.07.03), "Oscar Wilde le déchu" (30.07.03), "Wittgenstein le détraqué" (31.07.03), "Mandela l'endurant" (01.08.03). L'énoncé "Seuls contre tous" et le thème de la série pourraient être les signes précurseurs d'un retour du politique, d'une argumentation implicite etlou explicite en faveur du courage civil, de la liberté et de l'esprit de résistance, mais seuls trois des douze portraits ont des personnages politiques pour objet "Mandela l'endurant" est la seule personnalité politique contemporaine, or il n'est pas européen, mais africain. Sur les douze épithètes qui qualifient les personnages dans les titres, six impliquent un jugement de valeur négatif ("cynique", "insolent", "présomptueux", "imprécatrice", "déchu", "détraqué"), trois un jugement de valeur favorable ("opiniâtre", "combattante", "endurant"), deux une évaluation affective ("maudit", "incompris"), "Courbet le peintre" est neutre. Les voies de la repolitisation sont apparemment semées d'embûches... 